\title{
ASPECTOS EPIDEMIOLÓGICOS DA MENINGITE TUBERCULOSA EM MENORES DE 15 ANOS DE IDADE, NA GRANDE SÃO PAULO, BRASIL, 1982 - 1983*
}

\author{
Stella M.C. Nardy** \\ Roberto Brólio** \\ Marília Belluomini**
}

\begin{abstract}
NARDY, S.M.C. et al. Aspectos epidemiológicos da meningite tuberculosa em menores de 15 anos de idade, na Grande São Paulo, Brasil, 1982 - 1983. Rev. Saúde públ., S.Paulo. 23:117-27, 1989.

RESUMO: Foram estudadas algumas características epidemiológicas dos casos de meningite tuberculosa ocorridos em menores de 15 anos na Grande São Paulo (Brasil), nos anos de 1982 e 1983 . O levantamento dos dados foi realizado em fontes oficiais de informação, complementado pela visitação domiciliária. Foram identificados 126 casos, analisados segundo distribuição etária, sexo, fonte de contágio, vacinação $\mathrm{BCG}$, diagnóstico firmado, letalidade hospitalar, seqüelas e eventos ocorridos na seqüência do tratamento. Os resultados mostraram: demora no diagnóstico devido a prováveis fallas assistênciais, alta letalidade, identificação dos focos para a maioria dos casos. Houve dificuldade em avaliar a proteção conferida pela vacinação $B C G$ e na ocorrência de seqüelas, o grupo de menores de 5 anos foi o mais comprometido $(83,9 \%)$ enquanto que a maior letalidade ocorreu no grupo de 0 a 1 ano de idade $(43,1 \%)$. Houve $38,9 \%$ de cura; $33,3 \%$ de óbito; $15,1 \%$ de abandono e em $12,7 \%$ dos casos alguns permaneceram sob controle; e o restante era desconhecido pelo sistema de controle de notificações.
\end{abstract}

DESCRITORES: Tuberculose merígea. Epidemiologia.

\section{INTRODUÇÃO}

A meningite tuberculosa (MT) é a complicação mais severa de todas as formas da tuberculose, exigindo hospitalização para seu diagnóstico e tratamento inicial, uma vez que é virtualmente impossível a cura expontânea da doença. Foi durante muito tempo de prognóstico fatal, apresentando letalidade para o total de casos em todo o mundo.

E de consenso geral, que o declínio dessa letalidade começou a partir da descoberta dos quimioterápicos específicos para a tuberculose, em 1945, e atualmente é uma doença que pode ser considerada curável quando diagnosticada precocemente.

Escobar e col. ${ }^{3}$, em análise feita em 1975, alegam ser a letalidade menor nos países desenvolvidos, apresentando a doença graves problemas em áreas em desenvolvimento, decorrentes de desnutrição, más condições de vida e abandono de tratamento.

Para Alvarez ${ }^{1}$ quando a prevalência da tu- berculose é alta, a primo-infecção tuberculosa e a MT são freqüentes em idades mais baixas.

Editorial publicado no British Medical Journal ${ }^{20}$ mostra que a MT vem se tornando relativamente rara em países com elevados padröes médicos e sociais, podendo, com os modernos tratamentos, ser encontrada total recuperação sem seqüelas, se o tratamento foi iniciado antes da pessoa se tornar inconsiente.

No Brasil, em 1981, a incidência notificada de MT em menores de 5 anos de idade, apresentada por Gerhardt 5 , foi de 1,3 por 100.000 habitantes, ou seja, $23,6 \%$ do esperado que era 5,5 . Justifica o autor como sendo devido à alta cobertura de vacinação BCG em nosso meio. Acrescenta ainda que o sub-registro e a dificuldade para o diagnóstico podem influenciar esse resultado.

O Ministério da Saúde*** estima que a letalidade no país, por essa enfermidade, esteja em torno de $50 \%$, havendo "forte correlação entre o estágio da doença quando do início do tratamento e o prognóstico do paciente, sendo possível,

\footnotetext{
* Apresentado no $2^{\circ}$ Congresso Brasileiro de Pneumologia Pediátrica e $2^{\circ}$ Congresso Latino-Americano de Fíbrose Cístico (muco viscidose), Belo Horizonte, MG, 1987.

* Departamento de Epidemiologia da Faculdade de Saúde Pública da Universidade de São Paulo - Av. Dr. Arnaldo, 715 - 01255 - São Paulo, SP - Brasil.

*** Documento interno do Ministério da Saúde, sobre "Meningite Tuberculosa".
} 
entretanto, a diminuição da letalidade através do diagnóstico mais precoce".

No presente trabalho objetiva-se estudar casos diagnosticados de MT em menores de 15 anos de idade, na Grande São Paulo (GSP), nos anos de 1982 e 1983, analisando aspectos epidemiológicos e fatores que contribuem para a manutenção dessa enfermidade como problema de Saúde Pública, e oferecer subsídios para programas de controle da tuberculose.

\section{MATERIAL E MÉTODOS}

A população de estudo constituiu-se de casos de MT, em menores de 15 anos de idade, notificados na GSP em 1982 e 1983, acrescidos de casos diagnosticados e registrados em dois Hospitais especializados e sem notificação.

Os casos foram obtidos por meio de levantamento feito nos fichários do Centro de Investigação de Saúde da Secretaria da Saúde do Estado de São Paulo (CIS-SS) e nos Serviços de Arquivo Médico e Estatístico (SAME) de hospitais de São Paulo que internam o maior número de casos de MT no Estado de São Paulo: Hospital Emílio Ribas (HER) e Parque Hospitalar do Mandaqui (PHM).

A coleta de dados e informações sobre os casos foi realizada nesses locais, nos Centros de Saúde (CS) notificantes e em visitas domiciliárias (VD) feitas a todos os pacientes e/ou responsáveis.

\section{RESULTADOS E COMENTÁRIOS}

\section{Incidência da MT}

Observa-se acentuado decréscimo da MT em termos percentuais e em cocficientes por faixas etárias, nos dois anos do estudo (Tabela 1).
A comparação, por ano, revela para a faixa etária 0† 5 anos uma diminuição de casos de MT, para 1983, enquanto que para as demais faixas houve acréscimo de 0,65 para 0,83 e de 0,16 para 0,53 por 100.000 hab., em 1982 e 1983, respectivamente. Sobre o total houve um aumento do número de casos cujo coeficiente passou de 1,38, em 1982, para 1,47, em 1983.

Moraes ${ }^{10} \mathrm{em}$ estudo realizado em prontuários de pacientes residentes no Município de São Paulo e diagnosticados com MT no HER e no PHM, no período de 1955 a 1971, calculou coeficientes de morbidade por 100.000 habitantes dividindo a população em 3 grupos etários: $0 \mapsto 5 ; 5 \vdash 10$ e 10 e + anos de idade. Os coeficientes calculados para o primeiro grupo, nessa série histórica, apresentaram um decréscimo, passando de 8,91, em 1955, para 2,96, em 1971.

A Tabela 1 mostra que na GSP houve aumento da incidência de casos em menores de 15 anos, no ano de 1983, em relação a 1982, entretanto nada se pode concluir diante desses dados uma vez que seria necessário prolongar esses estudos por vários anos. Os coeficientes apresentados indicam que a MT, representa ainda um grave problema de Saúde Pública.

\section{Sexo e Idade}

Na Tabela 1 verificou-se que $76,2 \%$ dos casos de MT ocorreram na faixa etária de $0 \vdash 5$ anos. Por esse motivo, essa faixa etária, para o estudo das demais variáveis, foi redistribuída em grupos menores a fim de que se possa localizar as idades onde são encontrados os piores agravos.

Desta forma foram estudadas as variáveis sexo e idade (Tabela 2).

Analisando esta Tabela, na coluna do total, verifica-se que houve maior incidência entre $7 \mathrm{~m}-2$ anos, 56 casos equivalentes a $44,5 \%$ do total.

\section{TABELA 1}

Distribuição dos casos de MT diagnosticados na Grande São Paulo em menores de 15 anos segundo grupo etário e ano de diagnóstico, 1982 - 1983 (\% e coef. $\left.{ }^{*}\right)$

\begin{tabular}{|c|c|c|c|c|c|c|c|c|}
\hline \multirow{2}{*}{$\begin{array}{l}\text { Grupo } \\
\text { Etário }\end{array}$} & \multicolumn{3}{|c|}{1982} & \multicolumn{3}{|c|}{1983} & \multicolumn{2}{|c|}{ Total } \\
\hline & $\overline{\mathrm{N}^{\circ}}$ & $\%$ & Coef. & $\mathrm{N}^{\mathrm{Q}}$ & $\%$ & Coef. & $\mathrm{N}^{\mathrm{O}}$ & $\%$ \\
\hline $\begin{array}{r}0 \vdash 5 \\
5 \vdash 10 \\
10 \vdash 15\end{array}$ & $\begin{array}{r}49 \\
9 \\
2\end{array}$ & $\begin{array}{r}81,7 \\
15,0 \\
3,3\end{array}$ & $\begin{array}{l}2,93 \\
0,65 \\
0,16\end{array}$ & $\begin{array}{r}47 \\
12 \\
7\end{array}$ & $\begin{array}{l}71,2 \\
18,2 \\
10,6\end{array}$ & $\begin{array}{l}2,72 \\
0,83 \\
0,53\end{array}$ & $\begin{array}{r}96 \\
21 \\
9\end{array}$ & $\begin{array}{r}76,2 \\
16,7 \\
7,1\end{array}$ \\
\hline Total & 60 & 100,0 & 1,38 & 66 & 100,0 & 1,47 & 126 & 100,0 \\
\hline
\end{tabular}

* Por 100.000 habitantes (dados brutos populacionais obtidos na Fundação Sistema Estadual de Análise de Dados) MT - Meningite tuberculosa. 


\section{TABELA 2}

Distribuiçăo dos casos de MT diagnosticados na Grande São Paulo em menores de 15 anos, segundo sexo e grupo etário, 1982 - 1983 (№ e \%).

\begin{tabular}{lrrrr}
\hline $\begin{array}{l}\text { Grupo } \\
\text { Etário }\end{array}$ & \multicolumn{2}{c}{ Masc } & \multicolumn{2}{c}{ Fem. } \\
\hline $0 \vdash 4 \mathrm{~m}$ & 3 & 75,0 & 1 & 25,0 \\
$4 \vdash \mathbf{m}$ & 7 & 50,0 & 7 & 50,0 \\
$7 \vdash 12 \mathrm{~m}$ & 23 & 69,7 & 10 & 30,3 \\
$1 \mathrm{a}-2 \mathrm{a}$ & 14 & 60,9 & 9 & 39,1 \\
$2 \vdash 3$ & 7 & 77,8 & 2 & 22,2 \\
$3 \vdash 4$ & 5 & 62,5 & 3 & 37,5 \\
$4 \vdash 5$ & 5 & 100,0 & - & - \\
$5 \vdash 10$ & 14 & 66,7 & 7 & 33,3 \\
$10 \vdash 15$ & 5 & 55,6 & 4 & 44,4 \\
\hline Total & 83 & 65,9 & 43 & 34,1 \\
\hline
\end{tabular}

MT - Meningite Tuberculosa

Em trabalho publicado no Japão, Mori e $\mathrm{col}^{11}$. mostram que de 256 pessoas diagnosticadas com MT, no ano de 1979, naquele país, 43 (16,8\%) eram menores de 15 anos. Destes, 30 (70\%) pertenciam ao grupo etário 015 anos e entre eles 17 (40\%) possuiam idade menor de 1 ano.

Comparando os dados daqueles autores 11 com os apresentados no presente trabalho verifica-se que dos 126 casos estudados $96(76,2 \%)$ eram menores de 5 anos e entre estes $51(53,1 \%)$ estavam compreendidos na idade menor de um ano.

Tiriba e col. ${ }^{18}$, analisando 214 casos de MT atendidos no HER, no período de 1975 a 1979, encontraram para a população de estudo, 110 casos $(51,4 \%)$ na faixa etária $0 \vdash 5$ anos e entre esses, 46 $(41,8 \%)$ eram menores de um ano de idade.

Em face dos dados apresentados fica evidente a importância da MT como um sério problema de saúde não só pela alta incidência em crianças de baixa idade, mas também por se tratar de uma doença de caráter altamente letal e mutilante.

Estudando a variável sexo nota-se uma preponderância do sexo masculino sobre o feminino nas diferentes faixas etárias, apresentando em quase todas uma relaçào de 2:1 (Tabela 2). Esse fato não causa estranheza visto que na literatura pesquisada vários autores encontraram, também, nítida predominância no sexo masculino $1,2,6$, $11,14,16,18,22,23$.

\section{Fonte de Contágio}

A descoberta da fonte de contágio no ambiente intra ou extra domiciliar fornece elementos conclusivos para o estabelecimento do diagnóstico da MT.

Segundo Tiriba e col ${ }^{18}$, - "Deve-se habilidosamente procurar obter a confidência sobre turberculose familiar, pois dados epidemiológicos positivos associados a sinais nervosos reforçam a suspeita da doença específica".

$\mathrm{Na}$ anamnese da $\mathrm{MT}$, a investigação quanto à exposição a um foco tuberculoso é recomendada como de interesse vital por vários autores $8,11,23$.

\section{TABELA 3}

Distribuição dos casos de MT diagnosticados na Grande São Paulo em menores de 15 anos, por grupo etário, informação sobre fonte de contágio, 1982 - 1983 (Ne \%)

Fonte de Contágio

\begin{tabular}{|c|c|c|c|c|c|c|c|c|c|c|}
\hline \multirow{3}{*}{$\begin{array}{l}\text { Grupo } \\
\text { Etário }\end{array}$} & \multicolumn{8}{|c|}{ Fonte de Contágio } & \multirow{2}{*}{\multicolumn{2}{|c|}{ Total }} \\
\hline & \multicolumn{2}{|c|}{ Atual } & \multicolumn{2}{|c|}{ Antiga } & \multicolumn{2}{|c|}{ Desconhecida } & \multirow{2}{*}{\multicolumn{2}{|c|}{$\begin{array}{l}\text { Não informada } \\
N^{2} \%\end{array}$}} & & \\
\hline & $\mathrm{N}^{\circ}$ & $\%$ & $\mathrm{~N}^{\circ}$ & $\%$ & $\mathrm{~N}^{\circ}$ & $\%$ & & & $\mathrm{~N}^{\mathrm{O}}$ & $\%$ \\
\hline $0 \vdash 4 \mathrm{~m}$ & 3 & 75,0 & - & - & 1 & 25,0 & - & - & 4 & 100,0 \\
\hline $4 \vdash 7 \mathrm{~m}$ & 5 & 35,7 & - & - & 7 & 50,0 & 2 & 14,3 & 14 & 100,0 \\
\hline $7 \vdash 12 m$ & 19 & 57,6 & - & - & 11 & 33,3 & 3 & 9,1 & 33 & 100,0 \\
\hline $1 a-2 a$ & 11 & 478 & - & - & 7 & 30,4 & 5 & 21,7 & 23 & 100,0 \\
\hline $2 \vdash 3$ & 7 & 77,8 & - & - & 2 & 22,2 & - & - & 9 & 100,0 \\
\hline $3 \vdash 4$ & 4 & 50,0 & - & - & 3 & 37,5 & 1 & 12,5 & 8 & 100,0 \\
\hline $4 \vdash 5$ & 1 & 20,0 & - & - & 2 & 40,0 & 2 & 40,0 & 5 & 100,0 \\
\hline $5 \vdash 10$ & 6 & 28,6 & 5 & 23,8 & 7 & 33,3 & 3 & 14,3 & 21 & 100,0 \\
\hline $10 \vdash-15$ & 4 & 44,4 & 1 & 11,1 & 4 & 44,4 & - & - & 9 & 100,0 \\
\hline Total & 60 & 47,6 & 6 & 4,8 & 44 & 34,9 & 16 & 12,7 & 126 & 100,0 \\
\hline
\end{tabular}

MT - Meningite Tuberculosa 
Na Tabela 3, pelo levantamento de dados feito em VD foram consideradas: fonte atual - a referência a um ou mais contactos há menos de dois anos com doentes de tuberculose pulmonar, intra ou extra domiciliar; fonte antiga - citações de contactos ocorridos há mais de dois anos; fonte desconhecida - casos para os quais não se obteve resposta positiva referente a contactos com pessoas doentes no âmbito social; e não informada - casos para os quais não se conseguiu a informação por não ter sido realizada a VD ou se o foi não souberam esclarecer a questão.

A Tabela 3 mostra, para a população tomada como um todo, que em 60 casos $(47,6 \%)$ houve referência a foco atual; $6(4,8 \%)$ a foco antigo; em $44(34,9 \%)$ a resposta foi negativa quanto ao contato com foco atual ou antigo e, em $16(12,7 \%)$ não foi possível obter a informação.

Ressalte-se que em $50 \%$ dos casos houve referência a fontes de contágio atuais principalmente nos primeiros 5 anos de vida. Como essa referência atingiu, em sua grande maioria, esse grupo, admite-se que o grau de intimidade, a que o caso esteve exposto, tenha sido o responsável pelo desencadeamento da doença.

A informaçăo sobre fonte antiga somente foi presente para as idades acima de 5 anos o que sugere que estas crianças foram infectadas ou que adquiriram sua primo-infecção em idades mais baixas. Para o caso dos $34,9 \%$ que negaram a existência de contágio, não está excluída a possibilidade de contato com fontes desconhecidas.

\section{Vacinação BCG}

O estudo desta variável é importante uma vez que a "finalidade da vacinação é substituir a primo-infecção natural por bacilo virulento da tuberculose, perigoso por sua potência, por uma primo-infecção artificial e inofensiva ocasionada por bacilo não virulento, na esperança de que uma infecção artificial venha a contribuir, igualmente, para aumentar a resistência do indivíduo em face de uma infecção ulterior por bacilo virulento" 15 .

As divergências mundiais encontradas quanto à eficácia da vacinação BCG, desde sua descoberta, na França, por Calmet e Guérin, levaram em 1980, um grupo de estudiosos da OMS a fazer a seguinte recomendação: "Nos países com elevada prevalência da tuberculose, a vacinação com BCG deve ser realizada o mais cedo possível, visto que existem provas de que pode ter um papel valioso na prevenção de formas graves de tuberculose infantil tais como tuberculose miliar e meningite tuberculosa 12 .

Wunsh $F^{Q} 21$ procurou estimar a proteção contra a MT em crianças menores de 5 anos, residentes na GSP e diagnosticadas no HER e PHM, nos anos de 1981 a 1983 . Os resultados da investigação mostraram uma magnitude de proteçăo de $90,0 \%$ quando comparou casos e controles de vizinhança, concluindo que a vacina BCG é altamente eficaz para proteger crianças menores de 5 anos contra aquela manifestação da tuberculose.

$\mathrm{Na}$ execução da presente pesquisa a preocupação em localizar uma informação correta sobre antecedente vacinal, pelo BCG, entre os casos estudados, foi uma constante. Quando a informação não foi suficiente na $V D$, principalmente nos menores de 5 anos, procurou-se complementação nos CS e nos prontuários hospitalares.

$O$ antecedente positivo e negativo mostrado na Tabela 4, foi comprovado para 107 casos $(84,9 \%)$, pela verificação em cadernetas de vacinação e ou cicatriz vacinal. Para os restantes 19 casos $(15,1 \%)$ não foi possível obtê-la por óbito do paciente ou ausência do mesmo no momento da

\section{TABELA 4}

Distribuição dos casos de MT diagnosticados na Grande São Paulo em menores de 15 anos, por grupo etário, segundo antecedente vacinal por BCG, 1982 - 1983 ( ${ }^{\circ}$ e \%)

\begin{tabular}{|c|c|c|c|c|c|c|c|c|}
\hline $\begin{array}{l}\text { Grupo } \\
\text { Etário }\end{array}$ & \multicolumn{2}{|c|}{ Positivo } & \multicolumn{2}{|c|}{$\begin{array}{l}\text { Antecedente Vacinal } \\
\text { Negativo }\end{array}$} & \multicolumn{2}{|c|}{ Sem Inform. } & \multicolumn{2}{|c|}{ Total } \\
\hline $\begin{array}{l}0 \vdash 4 \mathrm{~m} \\
4 \vdash 7 \mathrm{~m} \\
7 \vdash 12 \mathrm{~m} \\
1 \mathrm{a} \vdash 2 \mathrm{a} \\
2 \vdash 3 \\
3 \vdash 4 \\
4 \vdash 5 \\
5 \vdash 10 \\
10 \vdash 15\end{array}$ & $\begin{array}{r}1 \\
7 \\
21 \\
10 \\
4 \\
4 \\
2 \\
8 \\
5\end{array}$ & $\begin{array}{l}25,0 \\
50,0 \\
63,7 \\
43,5 \\
44,4 \\
50,0 \\
40,0 \\
38,1 \\
55,6\end{array}$ & $\begin{array}{r}3 \\
5 \\
8 \\
10 \\
5 \\
3 \\
2 \\
7 \\
2\end{array}$ & $\begin{array}{l}75,0 \\
35,7 \\
24,2 \\
43,5 \\
55,6 \\
37,5 \\
40,0 \\
33,3 \\
22,2\end{array}$ & $\begin{array}{l}- \\
2 \\
4 \\
3 \\
- \\
1 \\
1 \\
6 \\
2\end{array}$ & $\begin{array}{c}- \\
14,3 \\
12,1 \\
13,0 \\
- \\
12,5 \\
20,0 \\
28,6 \\
22,2\end{array}$ & $\begin{array}{r}4 \\
14 \\
33 \\
23 \\
9 \\
8 \\
5 \\
21 \\
9\end{array}$ & $\begin{array}{l}100,0 \\
100,0 \\
100,0 \\
100,0 \\
100,0 \\
100,0 \\
100,0 \\
100,0 \\
100,0\end{array}$ \\
\hline Total & 62 & 49,2 & 45 & 35,7 & 19 & 15,1 & 126 & 100,0 \\
\hline
\end{tabular}

MT - Meningite Tuberculosa 
VD, ou porque nos prontuários procurados não havia referência à situação vacinal.

A Tabela 4 mostra, ainda, que do total de 126 crianças, $62(49,2 \%)$ haviam sido vacinadas pelo BCG e $45(35,7 \%)$ não tinham esse antecedente. Deixa também evidente que a maior percentagem de vacinados encontrava-se na faixa etária $7 \vdash 12$ meses $(63,7 \%)$. Os altos percentuais de nãovacinação, nas faixas etárias da população estudada, diferem bastante dos dados do Ministério da Saúde 19, que revelam para o Brasil, em 1981, uma cobertura vacinal de $81 \%$ para os menores de 15 anos e para os Estados do Rio de Janeiro e Såo Paulo, na idade menor de um ano, coberturas de, respectivamente, $96 \%$ e $99 \%$.

Mori e col. ${ }^{11}$ encontraram no estudo feito no Japão, entre 43 casos de MT estudados de menores de 15 anos, 8 (19\%) com antecedente vacinal positivo.

Delage e Dusseault ${ }^{2}$, no Hospital Santa Justina, em Montreal, encontraram 25 vacinados entre 79 casos de MT (31,7\%).

Para complementação de dados na presente pesquisa, procurou-se comprovar a data em que as 62 crianças haviam sido vacinadas e pesquisar entre elas a presença de foco tuberculoso domiciliar. Dos 48 casos com data comprovada, 27 informaram a presença de foco. Fazendo um confronto entre a data da vacinação com a data da descoberta de foco, foi observado que 12 casos $(44,4 \%)$ haviam sido vacinados antes da descoberta e 15 $(55,6 \%)$ o foram posteriormente. Este dado mostra a importância em se conhecer a situação de antecedente tuberculoso entre aqueles que se apresentam para vacinação $B C G$. A prática da vacinação indiscriminada, ou seja, sem PPD prévio, impossibilita a seleção dos reatores, que uma vez vacinados não se beneficiariam com o poder de proteção contra a MT. Este fato pode ter ocorrido entre os 15 casos mencionados acima, uma vez, que não é possível comprovar se a criança já estava infectada antes de ter sido vacinada.

\section{Dignóstico firmado}

Não foi objetivo do presente trabalho analisar os critérios adotados para que fossem firmados esses diagnósticos. Os dados apresentados na Tabela 5 revelam os diagnósticos transcritos das fichas e ou prontuários dos casos estudados, obtidos nos levantamentos realizados. Geralmente constava, naqueles registros, apenas 0 diagnóstico referente à MT ou um sinônimo qual seja meningo-encefalite por tuberculose ou neuro-tuberculose. Outras vezes, referiam uma associação com um processo de tuberculose pulmonar, tuberculose miliar e raramente com tuberculose extra Pulmonar, como tuberculose ossea ou ganglionar.

Pela Tabela 5 observa-se que o diagnóstico de MT, associado a outro processo tuberculoso, incide, de um modo geral, mais freqüentemente entre as crianças de até 4 anos. Apenas 5 crianças com mais de 5 anos tiveram a MT associada a outro processo tuberculoso, situação já largamente citada em trabalhos com populações semelhantes. É nítida a intercorrência da tuberculose miliar em casos de MT, no primeiro ano de

\section{TABELA 5}

Distribuição dos casos de $\mathrm{MT}^{*}$ diagnosticados na Grande São Paulo em menores de 15 anos, por grupo etário, segundo diagnóstico firmado, 1982 - 1983 (№ e \%)

\begin{tabular}{|c|c|c|c|c|c|c|c|c|c|c|}
\hline \multirow{2}{*}{$\begin{array}{l}\text { Grupo } \\
\text { Etário }\end{array}$} & \multicolumn{2}{|c|}{ MT } & \multicolumn{2}{|c|}{$\begin{array}{l}\mathrm{MT}+\mathrm{TBC} \\
\text { Pulmonar }\end{array}$} & \multicolumn{2}{|c|}{$\begin{array}{c}\text { MT }+ \text { TBC } \\
\text { Miliar }\end{array}$} & \multicolumn{2}{|c|}{$\begin{array}{c}\mathrm{MT}+\mathrm{TBC} \\
\text { Extra Pulmonar }\end{array}$} & \multicolumn{2}{|c|}{ Total } \\
\hline & $\mathrm{N}^{\mathrm{e}}$ & $\%$ & $\mathrm{~N}^{\circ}$ & $\%$ & $\mathrm{~N}^{\circ}$ & $\%$ & $\mathrm{~N}^{\circ}$ & $\%$ & $\mathrm{~N}^{\circ}$ & $\%$ \\
\hline $\mathrm{or}-4 \mathrm{~m}$ & 1 & 25,0 & 1 & 25,0 & 2 & 50,0 & - & - & 4 & 100,0 \\
\hline $4 \vdash 7 \mathrm{~m}$ & 6 & 42,8 & 4 & 28,6 & 4 & 28,6 & - & - & 14 & 100,0 \\
\hline $7 \vdash 12 \mathrm{~m}$ & 15 & 45,5 & 7 & 21,2 & 11 & 33,3 & - & - & 33 & 100,0 \\
\hline $1 a-2 a$ & 15 & 65,2 & 5 & 21,7 & 2 & 8,7 & 1 & 4,4 & 23 & 100,0 \\
\hline $2 \vdash 3$ & 6 & 66,7 & 2 & 22,2 & - & - & 1 & 11,1 & 9 & 100,0 \\
\hline $3 \vdash 4$ & 7 & 87,5 & 1 & 12,5 & - & - & - & - & 8 & 100,0 \\
\hline $4 \vdash 5$ & 5 & 100,0 & - & - & - & - & - & - & 5 & 100,0 \\
\hline $5 \vdash 10$ & 17 & 81,0 & - & - & 4 & 19,0 & - & - & 21 & 100,0 \\
\hline $10-15$ & 8 & 88,9 & - & - & 1 & 11,1 & - & - & 9 & 100,0 \\
\hline Total & 80 & 63,5 & 20 & 15,9 & 24 & 19,0 & 2 & 1,6 & 126 & 100,0 \\
\hline
\end{tabular}

MT - Meningite Tuberculosa

TBC - Tuberculose 
vida; das 24 crianças que tiveram esta associação, $17(70 \%)$ estavam na faixa etária de $0 \vdash 12$ meses. Neste mesmo grupo de idade a tuberculose pulmonar aparece associada à MT em $60 \%$.

Mais uma vez fica evidenciada a presença de focos de tuberculose ativa entre essas crianças, que uma vez expostas ao contágio não tiveram condições de superar a infecção dando oportunidade ao aparecimento da doença sob formas graves.

Alvarez e col. ${ }^{1}$ afirmam que a associaçăo da tuberculose miliar com a meningite varia de 1 a $79 \%$, independentemente da idade, mas freqüentemente não é reconhecida em vida. Citando Emery aqueles autores, revelam que em estudo realizado entre 63 crianças com MT, em $52(82,0 \%)$ foi constatado, por autópsia, tuberculose miliar.

\section{Alta hospitalar}

O tratamento da MT exige, inicialmente, um tempo de hospitalização pois o quadro sintomatológico requer uma atenção multiespecializada em termos de pediatria, neurologia, oftalmologia, fisioterapia e enfermagem. Uma vez superados os problemas mais urgentes, a continuaçãăo do tratamento pode ser realizada no domicilio ${ }^{17}$.

As altas hospitalares foram registradas em termos de : transferências para o CS para consolidação do tratamento; transferências para outro hospital, indicando casos que necessitavam continuar hospitalizados; transferência para ambulatórios por interesse do médico que atendeu na hospitalização; óbitos, indicando falência do tratamento e, conseqüentemente, a letalidade hospitalar, e finalmente, abandono do tratamento no próprio hospital, por alta a pedido.

Pelos dados da Tabela 6, observa-se que dos 126 casos de MT internados, apenas $50,8 \%$ saíram com alta para continuidade do tratamento em nível de CS. Os casos que aparecem como transferidos para outro hospital ou para ambulatório são casos gravíssimos exigindo continuidade de internação em hospitais especializados, ou seguimento no ambulatório do próprio hospital por interesse médico. Perfazem a totalidade de $30 \mathrm{ca}$ sos, ou seja, 23, $8 \%$. Mais uma vez fica evidenciada a gravidade da doença entre os menores de um ano, pois nesta faixa de idade ocorreu mais da metade do número de óbitos por MT na idade de até 15 anos, representando $33,3 \%$ de letalidade hospitalar. Para a população de estudo, como um todo, esse evento foi de $24,6 \%$. Esses dados podem significar um retardo no atendimento específico o que impediu a possibilidade de uma recuperação levando o doente a b́bito.

O único caso de abandono hospitalar registrado refere-se a um paciente que foi retirado do hospital pois os pais não acreditavam no tratamento recomendado.

\section{Seqüelas}

O registro de ocorrência das seqüelas quando das altas hospitalares, não foi uma constante nos prontuários examinados. Entretanto, julgando de interesse demonstrar qual o estado dos pacientes à saída do hospital onde se processou seu diagnóstico, aquela falha foi suprida, em parte, pelos relatos obtidos nas entrevistas domiciliares.

Reportando-se à Tabela 6, que indica 95 pacientes saídos do hospital com vida, foi feita a Tabela 7 que mostra, sobre seqüelas, como se encontravam aqueles pacientes segundo grupos etários.

\section{TABELA 6}

Distribuição dos casos de MT diagnosticados na Grande São Paulo em menores de 15 anos, por grupo etário, segundo tipo de alta hospitalar, $1982-1983\left(\mathrm{~N}^{\circ}\right.$ e \%)

\begin{tabular}{|c|c|c|c|c|c|c|c|c|c|c|c|c|}
\hline \multirow[t]{2}{*}{$\begin{array}{l}\text { Grupo } \\
\text { Etário }\end{array}$} & \multicolumn{2}{|c|}{$\begin{array}{l}\text { Transferência } \\
\text { Centro Saúde }\end{array}$} & \multicolumn{2}{|c|}{$\begin{array}{l}\text { Transferência } \\
\text { Hospital }\end{array}$} & \multicolumn{2}{|c|}{$\begin{array}{l}\text { Transferência } \\
\text { Ambulatório }\end{array}$} & \multicolumn{2}{|c|}{ Óbito } & \multicolumn{2}{|c|}{ Abandono } & \multicolumn{2}{|c|}{ Total } \\
\hline & $\mathrm{N}^{2}$ & $\%$ & No & $\%$ & $\mathrm{~N}^{\mathrm{e}}$ & $\%$ & $\mathrm{~N}^{2}$ & $\%$ & & $\%$ & $\mathrm{~N}^{\mathrm{e}}$ & $\%$ \\
\hline $\begin{array}{l}0 \vdash 4 \mathrm{~m} \\
4 \vdash 7 \mathrm{~m} \\
7 \vdash 12 \mathrm{~m} \\
1 \mathrm{a} \vdash 2 \mathrm{a} \\
2 \vdash 3 \\
3 \vdash 4 \\
4 \vdash 5 \\
5 \vdash 10 \\
10 \vdash 15\end{array}$ & $\begin{array}{r}2 \\
5 \\
14 \\
13 \\
8 \\
4 \\
1 \\
14 \\
3\end{array}$ & $\begin{array}{l}50,0 \\
35,7 \\
42,4 \\
56,5 \\
88,9 \\
50,0 \\
20,0 \\
66,7 \\
33,3\end{array}$ & $\begin{array}{l}- \\
2 \\
3 \\
3 \\
1 \\
2 \\
1 \\
1 \\
1\end{array}$ & $\begin{array}{r}14,3 \\
- \\
9,1 \\
13,0 \\
11,1 \\
25,0 \\
20,0 \\
4,7 \\
11,1\end{array}$ & $\begin{array}{l}1 \\
2 \\
4 \\
2 \\
- \\
- \\
1 \\
3 \\
3\end{array}$ & $\begin{array}{r}25,0 \\
14,3 \\
12,1 \\
8,7 \\
- \\
- \\
20,0 \\
14,3 \\
33,3\end{array}$ & $\begin{array}{r}1 \\
5 \\
11 \\
5 \\
- \\
2 \\
2 \\
3 \\
2\end{array}$ & $\begin{array}{l}25,0 \\
35,7 \\
33,3 \\
21,8 \\
- \\
25,0 \\
40,0 \\
14,3 \\
22,2\end{array}$ & $\begin{array}{l}- \\
- \\
- \\
- \\
- \\
- \\
- \\
-\end{array}$ & $\begin{array}{l}- \\
- \\
3,1 \\
- \\
- \\
- \\
- \\
-\end{array}$ & $\begin{array}{r}4 \\
14 \\
33 \\
23 \\
9 \\
8 \\
5 \\
21 \\
9\end{array}$ & $\begin{array}{l}100,0 \\
100,0 \\
100,0 \\
100,0 \\
100,0 \\
100,0 \\
100,0 \\
100,0 \\
100,0\end{array}$ \\
\hline Total & 64 & 50,8 & 14 & 11,1 & 16 & 12,7 & 31 & 24,6 & 1 & 0,8 & 126 & 100,0 \\
\hline
\end{tabular}

MT - Meningite Tuberculosa. 
Para 8 casos $(8,4 \%)$ não foi possível obter informação.

Entre os 62 casos que apresentaram seqüelas, estas estiveram presentes nas diferentes idades com uma variação de $38,9 \%$ a $100 \%$ dos casos, recaindo as maiores percentagens no grupo de $0 \vdash 5$ anos. Para os grupos subseqüentes a ausência de seqüelas sofre uma inversão, quando as maiores percentagens são encontradas no grupo com mais de 5 anos.

Lober ${ }^{7}$ realizou no "The Children's Hospital" em Sheffield, Inglaterra, estudo entre 100 crianças menores de 15 anos que sobreviveram a $\mathrm{MT}$, e concluiu que apesar da grande variedade de seqüelas o numero de crianças atingidas foi pequeno. $O$ autor encontrou em acompanhamento realizado durante 13 anos, 77 crianças subjetivamente normais e $23 \mathrm{com}$ vários tipos de seqüelas físicas e mentais.

Perfeito ${ }^{13}$ em estudo realizado no período de 1966 a 1971, em população com características semelhantes ao presente trabalho, encontrou entre 107 sobreviventes menores de 15 anos uma percentagem de $32,7 \%$ de seqüelas, equivalente a 35 casos, sendo que os demais $67,3 \%$ poderiam ser considerados normais após a doença.

\section{Avaliação da evolução dos casos}

Segundo Fitzsimons ${ }^{4}$, a sobrevivência do or-

\section{TABELA 7}

Distribuição dos casos de MT diagnosticados na Grande São Paulo em menores de 15 anos, por grupo etário que obtiveram alta hospitalar segundo presença ou não de seqüelas, $1982-1983$ (Nº e \%).

\begin{tabular}{|c|c|c|c|c|c|c|c|c|}
\hline \multirow{3}{*}{$\begin{array}{l}\text { Grupo } \\
\text { Etário }\end{array}$} & \multirow{2}{*}{\multicolumn{2}{|c|}{ Presente }} & \multicolumn{4}{|c|}{ Sequelas } & \multirow{2}{*}{\multicolumn{2}{|c|}{ Total }} \\
\hline & & & & & Sem & Inform. & & \\
\hline & $\mathrm{N}^{\circ}$ & $\%$ & $N^{\circ}$ & $\%$ & $N^{Q}$ & $\%$ & $N^{e}$ & $\%$ \\
\hline $0 \vdash 4 \mathrm{~m}$ & 3 & 100,0 & - & - & - & - & 3 & 100,0 \\
\hline $4 \vdash 7 \mathrm{~m}$ & 6 & 66,7 & 1 & 11,1 & 2 & 22,2 & 9 & 100,0 \\
\hline $7 \vdash 12 \mathrm{~m}$ & 15 & 68,2 & 5 & 22,7 & 2 & 9,1 & 22 & 100,0 \\
\hline $1 a-2 a$ & 14 & 77,8 & 3 & 16,7 & 1 & 5,5 & 18 & 100,0 \\
\hline $2 \vdash 3$ & 6 & 66,7 & 3 & 33,3 & - & - & 9 & 100,0 \\
\hline $3 \vdash 4$ & 5 & 83,3 & 1 & 16,7 & - & - & 6 & 100,0 \\
\hline $4 \vdash 5$ & 3 & 100,0 & - & - & - & - & 3 & 100,0 \\
\hline $5-10$ & 7 & 38,9 & 8 & 44,4 & 3 & 16,7 & 18 & 100,0 \\
\hline $10-15$ & 3 & 42,9 & 4 & 57,1 & - & - & 7 & 100,0 \\
\hline Total & 62 & 65,3 & 25 & 26,3 & 8 & 8,4 & 95 & 100,0 \\
\hline
\end{tabular}

MT - Meningite Tuberculosa

Nota: Eliminados os 31 casos que obtiveram alta hospitalar por óbito.

\section{TABELA 8}

Distribuição dos casos de MT diagnosticados na Grande São Paulo em menores de 15 anos, por grupo etário, segundo evento ocorrido na seqüência do tratamento, 1982 - 1983 ( $\mathrm{N}^{\circ}$ e \%)

\begin{tabular}{|c|c|c|c|c|c|c|c|c|c|c|c|c|c|c|c|c|}
\hline \multirow[t]{2}{*}{$\begin{array}{l}\text { Grupo } \\
\text { Etário }\end{array}$} & \multicolumn{2}{|c|}{ Cura } & \multicolumn{2}{|c|}{ Abandono } & \multicolumn{2}{|c|}{ Óbito } & \multicolumn{2}{|c|}{ Hospitalização } & \multicolumn{2}{|c|}{ Controle } & \multicolumn{2}{|c|}{$\begin{array}{l}\text { Tratamento } \\
\text { Desconhecido }\end{array}$} & \multicolumn{2}{|c|}{$\begin{array}{l}\text { Sem } \\
\text { Tratam. }\end{array}$} & \multicolumn{2}{|r|}{ Total } \\
\hline & $N^{\circ}$ & $\%$ & $\mathrm{~N}^{2}$ & $\%$ & $N^{2}$ & $\%$ & $N^{2}$ & $\%$ & $N^{2}$ & $\%$ & $N^{2}$ & $\%$ & $\mathrm{~N}^{2}$ & $\%$ & $\mathrm{~N}^{\circ}$ & $\%$ \\
\hline $\begin{array}{l}0 \vdash 4 \mathrm{~m} \\
4 \vdash 7 \mathrm{~m} \\
7 \vdash 12 \mathrm{~m} \\
1 \mathrm{a} \vdash 2 \mathrm{a} \\
2 \vdash 3 \\
3 \vdash 4 \\
4 \vdash 5 \\
5 \vdash 10 \\
10 \quad 15\end{array}$ & $\begin{array}{r}3 \\
5 \\
6 \\
10 \\
5 \\
4 \\
1 \\
9 \\
6\end{array}$ & $\begin{array}{l}75,0 \\
35,8 \\
18,2 \\
43,5 \\
55,6 \\
50,0 \\
20,0 \\
42,9 \\
66,7\end{array}$ & $\begin{array}{l}- \\
1 \\
6 \\
2 \\
3 \\
1 \\
1 \\
5\end{array}$ & $\begin{array}{r}7,1 \\
18,2 \\
8,7 \\
33,3 \\
12,5 \\
20,0 \\
23,8\end{array}$ & $\begin{array}{l}1 \\
7 \\
14 \\
8 \\
- \\
3 \\
2 \\
5 \\
2\end{array}$ & $\begin{array}{l}25,0 \\
50,0 \\
42,4 \\
34,8 \\
- \\
37,5 \\
40,0 \\
23,8 \\
22,\end{array}$ & $\begin{array}{l}- \\
- \\
- \\
- \\
- \\
- \\
- \\
-\end{array}$ & $\begin{array}{l}- \\
- \\
- \\
- \\
- \\
- \\
- \\
- \\
11,1\end{array}$ & $\begin{array}{l}- \\
\overline{1} \\
- \\
- \\
- \\
- \\
-\end{array}$ & $\begin{array}{l}- \\
-\overline{0} \\
- \\
- \\
- \\
- \\
- \\
-\end{array}$ & $\begin{array}{l}- \\
1 \\
4 \\
2 \\
1 \\
- \\
1 \\
2 \\
-\end{array}$ & $\begin{array}{r}- \\
7,1 \\
12,1 \\
8,7 \\
11,1 \\
- \\
20,0 \\
9,5 \\
-\end{array}$ & $\begin{array}{l}- \\
- \\
2 \\
1 \\
- \\
- \\
- \\
- \\
-\end{array}$ & $\begin{array}{l}- \\
- \\
6,1 \\
4,3 \\
- \\
- \\
- \\
-\end{array}$ & $\begin{array}{l}4 \\
14 \\
33 \\
23 \\
9 \\
8 \\
5 \\
21 \\
9\end{array}$ & $\begin{array}{l}100,0 \\
100,0 \\
100,0 \\
100,0 \\
100,0 \\
100,0 \\
100,0 \\
100,0 \\
100,0\end{array}$ \\
\hline Total & 49 & 38,9 & 19 & 15,1 & 42 & 33,3 & 1 & 0,8 & 1 & 0,8 & 11 & 8,7 & 3 & 2,4 & 126 & 100,0 \\
\hline
\end{tabular}

MT - Meningite Tuberculosa 
ganismo acometido por uma doença grave expressa a eficácia do tratamento realizado. Entre os fatores que afetam o prognóstico estão o grau de evolução da doença quando iniciou o tratamento, a idade do paciente e a associação com outro tipo de tuberculose.

$\mathrm{Na}$ avaliação do tratamento realizado, foram identificados os eventos ocorridos após o estabelecimento do diagnóstico em relação à idade dos doentes, independentemente do grau de evolução da entidade mórbida que os acometeu.

O tratamento realizado, basicamente, para todos os casos, foi o regime quimioterápico recomendado pela Divisão Nacional de Pneumologia Sanitária (DNPS) ${ }^{9}$.

A evolução dos casos mostrada na Tabela 8, refere-se à situaçăo final encontrada entre os 126 casos estudados. Estes dados foram obtidos após busca, praticamente individual, para cada caso, devido à inexistência de um efetivo entrosamento entre as Instituiçőes informantes. As informaçōes obtidas nas VD mostram que o percentual de cura foi de $38,9 \%$; o de abandono $15,1 \%$ e o de óbito $33,3 \%$. Voltaram à hospitalizaçăo $0,8 \%$, continuaram em controle $0,8 \%$ e estavam sem tratamento 2,4\% dos casos. Para $8,7 \%$ dos doentes nåo foi possível obter qualquer informação sobre a situação em que se encontravam quanto à doença. Em alguns casos a não existência de dados é devido a mudança de residência e a informação obtida na VD foi a de que aquelas crianças sobreviveram à doença, mas essa informação não foi localizada no Sistema.

Vale comentar o aumento sensível de óbitos e abandonos ocorridos na seqüência do tratamento, quando comparados com os tipos de alta hospitalar (Tabela 6). Chama também a atenção o número de altas por 6bito na faixa etária de 7 a 12 meses, tanto na fase de internação (Tabela 6) quanto na fase pós-hospitalar (Tabela 8).

Evidencia o presente trabalho a importância da MT como um problema de Saúde Pública, pois mesmo contando com recursos terapêticos e institucionais, somente $38,9 \%$ dos doentes lograram alcançar a cura, valendo inferir que estes recursos não estão sendo devidamente utilizados pela sociedade ou foram tardiamente procurados pela população.

\section{Relatos sobre o evento}

De acordo com os objetivos propostos são apresentados alguns relatos obtidos nas entrevistas domiciliares que ilustram as dificuldades encontradas pela população de análise, para o atendimento do caso e efetivação do diagnóstico.
Nesses resumos procura-se retratar com bastante fidedignidade os relatos feitos omitindo-se, entretanto, os nomes das entidades mencionadas.

A seleção dos mesmos obedecem o critério de maior significância, de acordo com a representatividade em número de casos semelhantes e 0 impacto causado, devido aos problemas apresentados.

CASO N 010 - A.A.S., feminino, 6 anos, vacinada, foco desconhecido; morando em habitação coletiva de $20 \mathrm{~m}^{2}$ com 5 pessoas, renda $\mathrm{fa}$ miliar entre um e dois salários mínimos e nível ocupacional não qualificado. Pessoa entrevistada: mãe.

"Chegou da escola com ânsia de vômito e febre, não sentia dor alguma, levei ao CS (a) e receitaram remédio para bichas; dei o remédio mas nada adiantou; daí levei ao PS (b), pois estava fraquinha, deram sôro e voltou para casa; não melhorou, levei novamente ao CS (a), aplicaram Benzetacil, pois disseram que era da garganta; năo melhorou, levei ao hospital (c) e receitaram mais remédios para a garganta inflamada; levei ao hospital (d), deram medicação para a febre, voltei com ela para casa mas entrou em delírio; levei ao hospital (c) e implorei que a internassem, mas receitaram Benzetacil e mandaram de volta; a criança não agüentava mais de fraqueza, levei mais 3 vêzes à farmácia onde deram medicamentos e nada de melhorar; daí levei ao hospital (f) e internaram com suspeita de infecção urinária; 3 dias após transferiram para o hospital (g) com suspeita de MT".

NOTA: Tempo de sintomas até internação: 20 dias; tempo de internação: 2 dias; conclusão: óbito; causa da doença: conhecida mas com foco não encontrado.

CASO N²047- C.A.S., 3 meses, não vacinado, foco domiciliar descoberto após diagnóstico; morando em barraco de favela de $10 \mathrm{~m}^{2}$ com 3 pessoas, renda familiar entre um e dois salários minimos, nível ocupacional não qualificado. Pessoa entrevistada: mãe.

"Assim que nasceu já tinha tosse sêca; quando estava com um mês levei ao hospital (a) onde ficou internado durante 8 dias; voltou para casa e depois de 3 dias apareceu um caroço na cabeça; voltei com ele ao hospital (a) e disseram que não era nada; o caroço continuou a crescer e a criança começou a emagrecer; levei ao hospital (b) e lá pediram para levar ao hospital (c), pois suspeita- 
vam de meningite e começo de turbeculose na cabeça."

NOTA: tempo de sintomas até internaçăo: 60 dias; tempo de internação: 1 dia; conclusão: óbito; causa da doença: desconhecida pela mãe, mas afirma estar em tratamento de tuberculose pulmonar no CS $(x)$ tendo sido descoberta sua doença após o falecimento da criança.

CASO No 128 - J.P.C., masculino, 1 ano e 4 meses, não vacinado, foco domiciliar; morando em casa sem acabamento, de $48 \mathrm{~m}^{2}$ com 8 pessoas, renda familiar maior que 5 salários mínimos e nível ocupacional não qualificado. Pessoa entrevistada: mãe.

"Começou apresentando febrinha, levei ao hospital (a) onde disseram que era da garganta e ia passar dentro de 3 a 4 dias com os antibióticos que receitaram; o problema não passou e voltei com a criança ao hospital (a) onde tiraram chapa, fizeram exame de sangue e urina e disseram que era pneumonia, receitando 3 doses de penicilina; a febre continuou e voltei novamente ao hospital (a) quando disseram que devia ser sarampo encubado. Voltei para casa esperando que estourasse o sarampo, dando apenas Novalgina, conforme orientação; 14 dias após voltei ao hospital (a) e disseram que era prisão de ventre, fizeram lavagem intestinal, deram Novalgina e mandaram de volta para casa, pois era feriado e não dava para dar maior assistência. Fiquei desesperada, e levei ao hospital (b), pois àquelas alturas o menino já estava com desnutrição, além da febre e vômitos, e precisava de internação urgente; como lá não tinha vaga encaminharam para o hospital (c), onde não quiseram atender pois o convênio que tínhamos era com o hospital (a). A enfermeira vendo a gravidade do caso e o meu desespero resolveu encaminhar para o hospital (d), onde foi atendido e internado com suspeita de meningite tuberculosa. Esteve internado durante 130 dias e quando saiu do hospital (d) continuou em controle no CS $(x)$ e na neurologia do hospital (d). Ele estava totalmente paralisado, tinha a cabeça crescida, não enxergava e tinha que ser operado para colocar a válvula, mas segundo o médico essa operação de nada valeria. Três dias antes de falecer, começou com problemas de deglutição, febre e vômitos; levei ao PS do hospital (a), mas já era tarde, apesar do balão de oxigênio, ele morreu".

NOTA: Tempo de sintomas até internação: 30 dias; tempo de internação: 130 dias; conclusão: óbito (após 9 meses de tratamento); causa da doença: desconhecida pela mãe, apesar de citar passado de tuberculose para si e seu marido, estando este, atualmente, em controle no CS (x).
Quanto à vacinação, nunca deixou vacinarem seus filhos pois "tem medo" de vacinas.

Várias conclusర̃es poderiam ser tiradas, ressaltando-se, entretanto, a procura de várias instituições de saúde para a solução do agravamento de problemas, decorrente provavelmente da falta de confiança no diagnóstico anterior e da insatisfação no atendimento obtido. Essa situação revela falhas assistenciais em prejuízo do diagnóstico e tratamento precoces.

Com a apresentação desses relatos espera-se poder contribuir para o aperfeiçoamento de uma estrutura organizada que possibilite o melhor atendimento futuro dos casos de MT, em nosso meio.

\section{CONCLUSÕES}

1 - A MT apresentou-se, nos anos de 1982 e 1983, como um grave problema de saúde pública, na GSP, para as idades de 0 a 15 anos, com evoluçōes mais desfavoráveis em crianças de baixa idade.

2 - O sexo masculino contribuiu com o maior número de casos de $\mathrm{MT}$ naqueles anos, e no total dos casos a idade entre 7 e 12 meses foi a mais atingida pela doença.

3- A proteção conferida pelo BCG não pode ser avaliada pela possibilidade de terem sido vacinadas crianças já infectadas pelo bacilo da tuberculose, sendo recomendável a realização de pesquisa de foco de infecção no momento da vacinação.

4- A MT associada a outras manifestações da tuberculose pulmonar incidiu, de modo preponderante, em crianças com idade até 5 anos.

5 - As seqüelas, desde distúrbios leves até os mais graves, tiveram uma ocorrência alta na população estudada tendo sido, porém, o grupo de $01-5$ anos o mais agredido pelas lesões.

6- As transferências para continuaçăo de tratamento em CS e os óbitos foram os dois motivos mais encontrados para a alta hospitalar. A letalidade hospitalar alta mostrou uma incapacidade de recuperação dos casos internados.

7 - A avaliação final do estudo mostra um índice baixo de cura em contraposição a um 
alto abandono e alta mortalidade, significando a não utilização correta dos recursos disponíveis no que se refere à prevenção, ao diagnóstico e tratamento da MT.

8 - Os relatos refletem que há um grande desconhecimento sobre a MT tanto por parte da população como por parte do pessoal de saúde. Com o diagnóstico correto feito a tempo e com uma boa orientação da população em como se comportar diante da doença, muito sofrimento humano seria poupado e muitas vidas preservadas.

NARDY, S.M.C. et al. [Epidemiological aspects of tuberculous meningits in children under 15 years of age, Greater S. Paulo, Brazil, 1982 - 1983]. Rev. Saúde públ., S.Paulo. 23:117-27, 1989.

ABSTRACT: Some epidemiological characteristics of cases of tuberculous meningitis which occurred in subjects under 15 years of age in Greater S. Paulo, S. Paulo State, Brazil, in 1982 and 1983, are studied. The cases were surveyed on the basis of official sources of information, complement by domiciliary visits. A hundred and twenty six (126) cases were identified and analysed by age, sex, source of contagion, BCG vaccination, confirmed diagnosis, hospital lethality, sequels and intercurrent events as part of the follow up. The results showed a delay in diagnosing the cases, possibly due to assistential failures; a high rate of lethality; identification of infectious focuses for the majority of the cases. There were difficulties in evaluating the protection provided by BCG vaccination and as regards sequels the most affected (83.9\%) were the under-fives while the greatest lethality rate (43.1\%) was found among the $0-1$ year-olds. At the end of the study there were $38.9 \%$ of cases of cure; $33.3 \%$ of death; $15.1 \%$ of withdrawal, and the remaining $12.7 \%$ some were still under control and others unknown to the system of notification.

KEYWORDS: Tuberculosis, meningeal, occurrence.

\section{REFERÊNCIAS BIBLIOGRÁFICAS}

1. ALVAREZ, S. \& Mc CABE, W. Extrapulmonary tuberculosis revisited: a review of experience at Boston City and other hospital. Medicine, 63:25-55, 1984.

2. DELAGE, G. \& DUSSEAULT, M. Tuberculous meningitis in children: a restrospective study of 79 patients with an analysis of prognostic factors. Canad. med. Ass. J., 120: 305-9, 1979.

3. ESCOBAR, J.A. et al. Mortality from tuberculous meningitis reduced by steroid therapy. Pediatrics, 56: 1050-5, 1975.

4. FITZSIMONS, J.M. Tuberculous meningitis: a follow up study on 198 cases. Tubercle, 44: 87-102, 1963.

5. GERHARDT, G. et al. Aspectos da epidemiologia da tuberculose no Brasil. J. Pneumol., 8 (suppl.) : 129, Nov. 1982.

6. HIRMAN, A.R. Tuberculous meningitis at Cleveland Metropolitan General Hospital. Amer Rev. resp. Dis., 95: 670-73, 1967.

7. LORBER, J. Long-term follow-up of 100 children who recovered from tuberculous meningitis. Pediatrics, 28: 778-91, 1961.
8. MARTINS, T.S. et al. Meningite tuberculosa: resultados de uma pesquisa operacional. J. bras. Med., 48 (4): 3440, 1985.

9. MNISTÉRIO DA SAÚtDE. Secretaria Nacional de Programas de Saúde. Divisào Nacional de Pneumologia Sanitária. Manual de normas para controle da tuberculose. $2^{4}$ ed. Brasilia. Centro de Documentação do Ministério da Saúde, 1984. (Série A. Normas e Manuais Técnicos, 13).

10. MORAES, J.C. Risco de infeç̧ão tuberculosa e incidência da meningite tuberculosa. São Paulo, 1977. [Dissertação de Mestrado - Departamento de Medicina Preventiva da Faculdade de Medicina da USP].

11. MORI, T. et al. La meningitis tuberculosa del niño en Japon. Bol. Un. int. Tuberc., 59: 201, 1984.

12. ORGANZACION MUNDIAL DE LA SALUD. Grupo de Estudios sobre Políticas de Vacunacion con BCG, Ginebra, 1980. Informe. Ginebra, 1980. (Série de Informes técnicos, 652).

13. PERFEITO, J.B. Contribuição para o estudo da tuberculose da criança, complicada com meningo-encefalite tuberculosa aplicando diferentes esquemas de tratamento. 
São Paulo, 1976. [Tese de Livre-Docência - Departamento de Clínica Médica da PUC].

14. PERFEITO, J.B. \& ASSIS, J.L. Meningo-encefalite tuberculosa. In: Veronesi, R. Doenças infecciosas e parasitárias. 7 ed. Rio de Janeiro, Guanabara - Koogan, 1982. p. 517-27.

15. SUTHERLAND, I Eficácia e duração da proteçâo conferida pela vacina BCG. Cad. int. Tuberc., (3), 1968. [Tradução - mimeogrado].

16. TARANTINO, A.B. et al. Meningite tuberculosa. In: Tarantino, A.B. Doenças pulmonares. Rio de Janeiro, Guanabara-Koogan, 1976. p. 383-5.

17. TIRIBA, A.C. Tuberculose volta a atacar. Folha de Säo Paulo, S.Paulo, 7 dez. 1980.

18. TIRIBA, A.C. et al. Meningite tuberculosa: falhas assistenciais em prejuízo do diagnóstico e tratamento precoces. Rev. Ass. méd. bras., 26: 248-52, 1980.
19. TUBERCULOSE. RADIS-Tema, Rio de Janeiro, 3(1) dez. 1982.

20. TUBERCULOUS meningitis in children [Editorial]. Brit. med.J., 1: 1-2, 1971.

21. WUNSCH Fo., V. Os estudos de caso-controle na avaliaçào da eficácia das vacinas: a eficácia da vacina BCG. São Paulo, 1985. [Dissertação de Mestrado - Departamento de Medicina Preventiva da Faculdade de Medicina da USP].

22. ZARABI, M. et al. The chest roentgenogram in the early diagnosis of tuberculous meningitis in children. Amer. J. Dis. Child., 121: 389-92, 1971.

23. ZERIHUN, G. \& ESSCHER, E. Ten years experience of tuberculous meningitis in children. Ethiop. med.J., 22: 49-54, 1984.

Recebido para publicação em $1 / 9 / 1988$ Reapresentado em 10/2/1989 Aprovado para publicação em 13/2/1989 